K. Kantarci, MD, MSc

R. Avula, PhD

M.L. Senjem, MS

A.R. Samikoglu

B. Zhang, MD

S.D. Weigand, MS

S.A. Przybelski

H.A. Edmonson, PhD

P. Vemuri, $\mathrm{PhD}$

D.S. Knopman, MD

T.J. Ferman, $\mathrm{PhD}$

B.F. Boeve, MD

R.C. Petersen, $\mathrm{MD}, \mathrm{PhD}$

C.R. Jack, Jr., MD

Address correspondence and reprint requests to Dr. Kejal Kantarci, Mayo Clinic, 200 First Street SW, Rochester, MN 55905 kantarci.kejal@mayo.edu

Supplemental data at www.neurology.org

\section{Dementia with Lewy bodies and Alzheimer disease}

\author{
Neurodegenerative patterns characterized by DTI
}

\section{ABSTRACT}

Objective: To identify the patterns of diffusivity changes in patients with dementia with Lewy bodies (DLB) and Alzheimer disease (AD) and to determine whether diffusion tensor MRI (DTI) is complementary to structural MRI in depicting the tissue abnormalities characteristic of DLB and AD.

Methods: We studied clinically diagnosed age-, gender-, and education-matched subjects with DLB $(n=30)$, subjects with AD ( $n=30)$, and cognitively normal $(C N)$ subjects $(n=60)$ in a case-control study. DTI was performed at 3T with a fluid-attenuated inversion recovery-based DTI sequence that enabled cortical diffusion measurements. Mean diffusivity (MD) and gray matter (GM) density were measured from segmented cortical regions. Tract-based diffusivity was measured using color-coded fractional anisotropy (FA) maps.

Results: Patients with DLB were characterized by elevated MD in the amygdala and decreased FA in the inferior longitudinal fasciculus (ILF). ILF diffusivity was associated with the presence of visual hallucinations ( $p=0.007$ ), and amygdala diffusivity was associated with Unified Parkinson's Disease Rating Scale ( $r=0.50 ; p=0.005)$ in DLB. In contrast, patients with AD were characterized by elevated MD in the medial temporal, temporal, and parietal lobe association cortices and decreased FA in the fornix, cingulum, and ILF. Amygdala diffusivity was complementary to GM density in discriminating DLB from $\mathrm{CN}$; hippocampal and parahippocampal diffusivity was complementary to GM density in discriminating $A D$ from $C N$.

Conclusion: Increased amygdalar diffusivity in the absence of tissue loss in dementia with Lewy bodies (DLB) may be related to microvacuolation, a common pathology associated with Lewy body disease in the amygdala. Diffusivity measurements were complementary to structural MRI, demonstrating that measures of diffusivity on diffusion tensor MRI are valuable tools for characterizing the tissue abnormalities characteristic of Alzheimer disease and DLB. Neurology ${ }^{\circledR}$ 2010;74:1814-1821

\section{GLOSSARY}

$\mathbf{A D}=$ Alzheimer disease; $\mathbf{C N}=$ cognitively normal; $\mathbf{D L B}=$ dementia with Lewy bodies; $\mathbf{D T I}=$ diffusion tensor $\mathrm{MRI} ; \mathbf{F A}=$ fractional anisotropy; FDR = false discovery rate; FLAIR = fluid-attenuated inversion recovery; $\mathbf{G M}=$ gray matter; ILF = inferior longitudinal fasciculus; LB = Lewy body; $\mathbf{M D}=$ mean diffusivity; RBD = REM sleep behavior disorder; $\mathbf{R O I}=$ region of interest; $\mathbf{S L F}=$ superior longitudinal fasciculus; TE = echo time; TI = inversion time; TR = repetition time; UPDRS = Unified Parkinson's Disease Rating Scale; WM = white matter

Dementia with Lewy bodies (DLB) is the second most common cause of neurodegenerative dementia after Alzheimer disease (AD). ${ }^{1}$ Although patients with Lewy body (LB) pathology typically have some $\mathrm{AD}$ pathology, ${ }^{2}$ noninvasive imaging markers that can distinguish the contribution of these different pathologies to the dementia syndrome may be useful for the differential diagnosis and may provide insight into the pathologic mechanisms underlying these disorders.

Diffusion tensor MRI (DTI) provides information on the integrity of tissue microstructure. Mean diffusivity (MD) in the brain increases with the degeneration of structural barriers that restrict the Brownian motion of water molecules such as myelin, cell membranes, and intracellular or-

From the Department of Radiology (K.K., R.A., M.L.S., A.R.S., B.Z., H.A.E., P.V., C.R.J.), Department of Health Sciences Research, Division of Biostatistics (S.D.W., S.A.P.), and Department of Neurology (D.S.K., B.F.B., R.C.P.), Mayo Clinic, Rochester, MN; Department of Radiology (B.Z.), Affiliated Drum Tower Hospital of Nanjing University Medical School, Nanjing, China; and Department of Psychiatry and Psychology (T.J.F.), Mayo Clinic, Jacksonville, FL.

Study funding: Supported by NIH (K23 AG030935, P50 AG16574/P1, R01 AG11378, R01 AG015866, U01 AG 06786). Support for several investigators was provided by the Robert H. and Clarice Smith and Abigail Van Buren Alzheimer's Disease Research Program of the Mayo Foundation and the NIH Construction Grant (NIH C06 RR018898).

Disclosure: Author disclosures are provided at the end of the article. 
ganelles. Directionality of diffusion along the axonal projections measured with fractional anisotropy (FA) decreases with degeneration of the white matter (WM). ${ }^{3}$ Diffusion MRI studies in $\mathrm{AD}$ have consistently found elevated $\mathrm{MD}$ in the hippocampus and decreased FA in the main limbic pathways connecting to the hippocampus. ${ }^{4-12}$ Less is known about the DTI changes in DLB. The few studies in DLB report conflicting findings, ranging from widespread diffusivity changes to no alteration in diffusivity. ${ }^{13-15}$ Furthermore, whether DTI would provide additional information over structural MRI in characterizing the cortical degeneration in $\mathrm{AD}$ and DLB is unknown.

Our primary objective was to identify the disease-specific patterns of diffusivity changes that are associated with alterations in tissue integrity in AD and DLB. Our secondary objective was to determine whether tissue diffusivity measurements are complementary to structural MRI in characterizing the neurodegenerative changes in $\mathrm{AD}$ and $\mathrm{DLB}$.

METHODS Subjects. During June 2007 to March 2009, 30 consecutive patients with DLB who met the Third Consortium criteria for probable $\mathrm{DLB}^{16}$ were recruited from the Mayo Clinic AD Research Center to participate in the MRI study. From the same source, we identified 30 patients with $\mathrm{AD}$ who met the National Institute of Neurological and Communicative Disorders and Stroke-Alzheimer's Disease and Related Disorders Association criteria for probable AD and were propensity matched to patients with DLB on age, gender, education, and Clinical Dementia Rating Sum of Boxes, which provided a general equivalence of functional dementia severity between the 2 patient groups. ${ }^{17}$ We identified 60 subjects with CN from the AD Research Center and Mayo Clinic Study of Aging, which is a longitudinal population-based cohort. Subjects with $\mathrm{CN}$ were propensity matched to patients with DLB (2:1) on age, gender, and education. Exclusion criteria were 1) presence of structural abnormalities that could cause cognitive impairment or dementia and 2) concurrent illnesses or treatments interfering with cognitive function other than AD or DLB. Patients who met the clinical criteria for both AD and DLB $(n=6)$ were also excluded in order to achieve homogeneity in clinical classification across the patient groups. This study was approved by the Mayo Institutional Review Board, and informed consent for participation was obtained from every subject or an appropriate surrogate.

The presence of clinical features of DLB was recorded using the following criteria: 1) severity of parkinsonism was rated with the Unified Parkinson's Disease Rating Scale (UPDRS); 2) visual hallucinations are fully formed, occurring on more than one occasion and not attributable to medical factors (e.g., infection, postoperative confusion), medications, or advanced dementia; 3) fluctuations are considered present if the patient scored 3 or 4 on the Mayo Fluctu- ations Questionnaire ${ }^{18}$; 4) patients with probable REM sleep behavior disorder (RBD) met the International Classification of Sleep Disorders-II diagnostic criteria B for RBD. ${ }^{19}$

MRI acquisition. MRI examinations were performed at 3 Tesla using an 8-channel phased array coil (GE, Milwaukee, WI) and parallel imaging with an acceleration factor of 2. A 3-dimensional high-resolution magnetization-prepared rapid gradient echo acquisition with repetition time (TR)/echo time (TE)/inversion time $(\mathrm{TI})=7 / 3 / 900 \mathrm{msec}$, flip angle 8 degrees, in plane resolution of 1.0 $\mathrm{mm}$, and a slice thickness of $1.2 \mathrm{~mm}$ was performed for anatomic segmentation and labeling. DTI was performed with an echoplanar imaging fluid-attenuated inversion recovery (FLAIR) sequence with TR/TI/TE $=8,800 / 2,200 / 60 \mathrm{msec}, 21$ diffusion-sensitive gradient directions $\left(\mathrm{b}=1,000 \mathrm{msec} / \mathrm{mm}^{2}\right)$, an in plane resolution of 2.96 $\mathrm{mm}$, and a slice thickness of $3.3 \mathrm{~mm}$. MD is significantly higher in $\mathrm{CSF}$ than the brain tissue. We avoided the partial volume averaging of cortical tissue with the higher diffusivity CSF signal by suppressing it with FLAIR DTI. ${ }^{20}$ Unlike the more standard T2-based DTI sequences, this FLAIR-based approach enabled reliable measures of cortical MD.

MRI analysis. Twenty-one diffusion-weighted images were registered to the non-diffusion-weighted FLAIR b0 volume with affine registration using 12 degrees of freedom in automated image registration software. ${ }^{21}$ Maps of MD, FA, and color-coded FA were generated using the DTIStudio software. ${ }^{22}$

The automated anatomic labeling atlas ${ }^{23}$ was modified inhouse to contain the following labeled regions of interest (ROI): amygdala, hippocampus, parahippocampal gyrus, posterior cingulate gyrus, and precuneus. These ROIs were chosen based on previous reports showing diffusivity changes in these regions in patients with $\mathrm{AD}$ and patients with DLB and the distribution of the neurodegenerative pathology in $\mathrm{AD}$ and DLB. Precentral and postcentral gyrus region was chosen as a reference ROI, because the primary sensory-motor cortex is on average the least pathologically affected cortical region in both $\mathrm{AD}$ and $\mathrm{LB}$ disease. Remaining cortical regions were grouped into lobar ROIs (i.e., frontal, parietal, occipital and temporal lobes). We did not analyze the basal forebrain and dorsal mesopontine nuclei in which we found decreased GM volumes in a previous study, ${ }^{24}$ because of the magnetic susceptibility artifacts and distortions in the basal forebrain and brainstem regions on EPI DTI images. All analyses were performed on the cortical GM.

The modified atlas was used to parcellate the GM, WM, and CSF segmented images into ROIs in each subject's T1 image space. The T1-weighted MRI volume of each subject was warped to his or her own FLAIR b0 image using an in-house modified version of the high dimensional warping toolbox in SPM5. The resulting deformation was applied to the subject-specific atlas, as well as to the native space segmented images, in order to warp the atlas labels as well as the segmented images to the subject's native FLAIR b0 image space. Atlas-based parcellation of MD maps into ROIs was therefore performed in subject FLAIR b0 image space. Statistics on MD values were extracted from each labeled cortical GM ROI in the atlas. Voxel-wise GM MD differences between groups were assessed in SPM5 and displayed at a significance value of $p<0.001$, corrected for multiple comparisons using false discovery rate (FDR).

WM tract-based analysis of MD and FA was performed by manual placement of ROIs on the color-coded FA maps using Analyze (Mayo Clinic, Rochester, MN) software by a radiologist (B.Z.) who was blinded to the clinical diagnosis. Anatomic landmarks were used when placing the ROIs, which were transferred to the FLAIR b0 images to ensure CSF spaces and WM hyperin- 


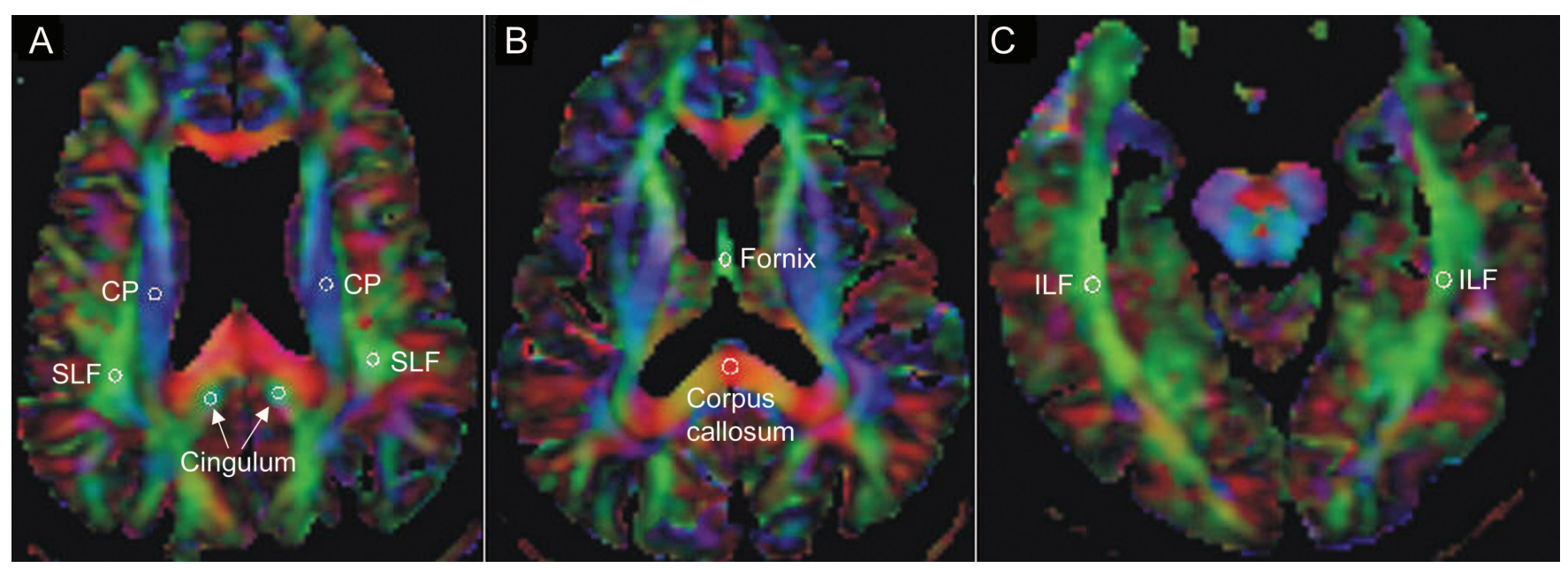

Corticopontine (CP), superior longitudinal fasciculus (SLF), and cingulum bundle ROls (A); corpus callosum and fornix ROIs (B); and inferior longitudinal fasciculus (ILF) ROIs (C) are shown.

tensities were avoided. We analyzed the major WM tracts including the fornix, corticopontine tracts, inferior longitudinal fasciculus, superior longitudinal fasciculus (SLF), cingulum bundles, and the corpus callosum (figure 1).

Statistical analysis. Because neither AD nor DLB pathologies differentially involve either hemisphere, right and left hemispheric values were averaged for statistical analysis. We performed a $\chi^{2}$ test of differences in gender distribution across groups and used a Kruskal-Wallis test to evaluate whether there were systematic differences in age and education across groups. We compared subjects with DLB and subjects with $\mathrm{AD}$ on numeric cognitive scores using the Wilcoxon rank-sum/MannWhitney $U$ test. We used univariate and bivariate binary logistic regression to evaluate pairwise group differences on each imaging measure at each ROI. Spearman rank-order correlation test was used to investigate the correlations between the imaging measurements and continuous clinical variables. We did not adjust $p$ values to account for multiple comparisons. ${ }^{25}$ However, to allow the reader to adjust $p$ values as desired, we report the actual values in the text or in figures e-1, e-2, and e-3 on the Neurology ${ }^{\circledR}$ Web site at www.neurology.org.

RESULTS Characteristics of the study subjects are listed in table 1 . Owing to the propensity matching procedure, $\mathrm{AD}, \mathrm{DLB}$, and $\mathrm{CN}$ groups had similar

Table 1 Characteristics of the study group

\begin{tabular}{|c|c|c|c|c|}
\hline Characteristics & $\mathrm{CN}^{\mathrm{a}}(\mathrm{n}=60)$ & DLB $(n=30)$ & $A D(n=30)$ & p Value ${ }^{b}$ \\
\hline Ratio of women to men (\% women) & $10: 50(17)$ & $4: 26(13)$ & $7: 23(23)$ & 0.58 \\
\hline Age, y, median (min, max) & $73(54,86)$ & $71(55,85)$ & $74(48,89)$ & 0.17 \\
\hline Education, y, median (min, max) & $14(8,20)$ & $14(8,20)$ & $16(8,20)$ & 0.55 \\
\hline $\begin{array}{l}\text { Short Test of Mental Status, median (min, } \\
\text { max) }\end{array}$ & $36(28,38)$ & $27(14,34)$ & $24(5,33)$ & 0.35 \\
\hline $\begin{array}{l}\text { Clinical Dementia Rating Sum of Boxes, } \\
\text { median (min, max) }\end{array}$ & $0(0,0)$ & $5(0,10)$ & $4(2,10)$ & 0.34 \\
\hline $\begin{array}{l}\text { Global Deterioration Scale, median (min, } \\
\text { max) }\end{array}$ & - & $4(3,6)$ & $4(3,6)$ & 0.32 \\
\hline $\begin{array}{l}\text { Unified Parkinson's Disease Rating Scale, } \\
\text { median (min, max) }\end{array}$ & - & $8(0,31)$ & $0(0,5)$ & $<0.001$ \\
\hline \multicolumn{5}{|l|}{$\begin{array}{l}\text { Ratio of those with vs without clinical } \\
\text { feature ( } \% \text { with feature) }\end{array}$} \\
\hline Fluctuations & - & $17: 10(63)$ & 3:21 (12) & $<0.001$ \\
\hline Hallucinations & - & $18: 10(64)$ & $0: 24(0)$ & $<0.001$ \\
\hline Probable REM sleep behavior disorder & - & $28: 0(100)$ & $7: 23(23)$ & $<0.001$ \\
\hline
\end{tabular}

Abbreviations: $\mathrm{AD}=$ Alzheimer disease; $\mathrm{CN}=$ cognitively normal; $\mathrm{DLB}=$ dementia with Lewy bodies.

a CN group not evaluated for the Global Deterioration Scale, Unified Parkinson's Disease Rating Scale, fluctuations, hallucinations, and REM sleep behavior disorder.

b From $\chi^{2}$ test for gender, fluctuations, hallucinations, and REM sleep behavior disorder; Kruskal-Wallis test for age and education; Wilcoxon rank-sum test of DLB vs AD for Global Deterioration Scale, Short Test of Mental Status, Clinical Dementia Rating Sum of Boxes, and Unified Parkinson's Disease Rating Scale. 
demographic features. Functional dementia severity measured with GDS and Clinical Dementia Rating Sum of Boxes was not different among patients with $\mathrm{AD}$ and patients with DLB.

Atlas-based ROI analysis in patients with DLB revealed elevated cortical MD only in the amygdala $(p=0.02)$, with normal MD in all of the other regions compared to the $\mathrm{CN}$ group (figure e-1). We found reduced cortical GM density in the hippocampus and temporal lobe in patients with DLB compared to $\mathrm{CN}$ (figure e-2). Voxel-based analysis of the cortical MD did not reveal any changes in patients

Figure 2 Voxel-based analysis of the gray matter diffusivity using SPM5
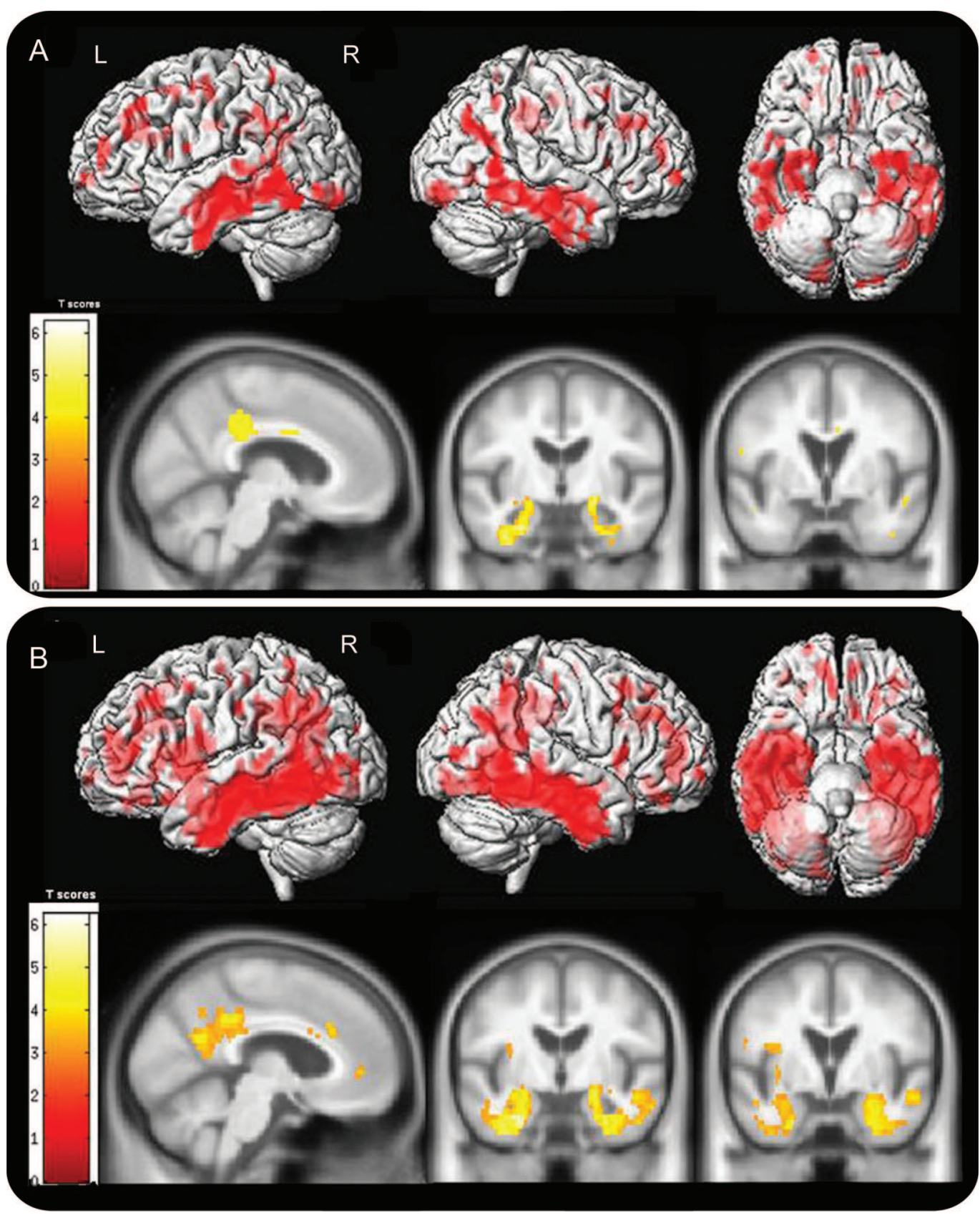

The panels show elevated diffusivity in the Alzheimer disease (AD) compared to the dementia with Lewy bodies (DLB) (A) and cognitively normal group (B) in red and yellow (false discovery rate [FDR] correction $p<0.001$ ). Surface render projections show that the diffusivity is elevated predominantly temporal and parietal cortex in the subjects with AD (denoted by red pixels) compared to the DLB and cognitively normal group, in agreement with the typical distribution of the neurofibrillary pathology in AD. The cross-sections demonstrate the increased diffusivity (yellow pixels) in the posterior cingulate gyrus and precuneus and the medial temporal lobe including hippocampus, parahippocampal gyrus in AD compared to patients with DLB and cognitively normal subjects. Whereas amygdala diffusivity is elevated in AD compared to the cognitively normal subjects, no difference in amygdala diffusivity is present among subjects with $A D$ and subjects with DLB, because the amygdala diffusivity is increased in both $A D$ and DLB. 
with DLB compared to the matched CN group at a $p$ value threshold of $<0.001$ and FDR correction for multiple comparisons. Higher MD $(r=0.5 ; p=$ $0.005)$ in the amygdala was associated with higher UPDRS scores in patients with DLB. No associations were identified between the amygdalar MD vs presence of visual hallucinations and fluctuations in patients with DLB $(p>0.05)$.

Patients with AD had elevated MD and decreased GM density in the hippocampus, parahippocampal gyrus, amygdala, temporal and parietal lobe association cortices, posterior cingulate gyrus, and precuneus cortices compared to the CN and DLB subjects $(p<0.05)$ (figure e-1). In the frontal lobe, occipital lobe, precentral and postcentral cortices, we identified reductions in GM density in patients with $\mathrm{AD}$ compared to the DLB and $\mathrm{CN}$ groups $(p<0.05)$ with a trend of elevation in MD in the frontal and occipital lobe cortices but not in the precentral and postcentral cortices $(p>0.05)$ (figure e-2). The pattern of MD elevation in patients with $\mathrm{AD}$ compared to the $\mathrm{CN}$ and DLB groups is demonstrated by voxel-based analysis $(p<0.05$ with FDR) in figure 2.

We investigated the additive value of cortical MD measurements over the cortical GM density measure-

\begin{tabular}{|c|c|c|c|}
\hline \multicolumn{4}{|c|}{$\begin{array}{l}\text { Median (interquartile range) for } 4 \text { white matter tract DTI } \\
\text { measurements by group within ROI }\end{array}$} \\
\hline Tract & Group & FA & MD \\
\hline \multirow[t]{3}{*}{ ILF } & $\mathrm{CN}$ & $0.57(0.54,0.6)$ & $798(762,827)$ \\
\hline & DLB & $0.54(0.51,0.57)^{a}$ & $795(774,827)$ \\
\hline & $A D$ & $0.54(0.51,0.57)^{a}$ & $826(791,842)^{a}$ \\
\hline \multirow[t]{3}{*}{ Fornix } & $\mathrm{CN}$ & $0.66(0.61,0.71)$ & $1,010(971,1049)$ \\
\hline & DLB & $0.67(0.63,0.73)$ & $992(949,1072)$ \\
\hline & $A D$ & $0.63(0.58,0.67)^{b}$ & $1,078(1005,1152)^{a}$ \\
\hline \multirow[t]{3}{*}{ Posterior cingulum } & $\mathrm{CN}$ & $0.6(0.57,0.63)$ & $723(691,740)$ \\
\hline & DLB & $0.6(0.57,0.63)$ & $701(679,739)$ \\
\hline & $A D$ & $0.55(0.52,0.6)^{b}$ & $740(731,757)^{c}$ \\
\hline \multirow[t]{3}{*}{ SLF } & $\mathrm{CN}$ & $0.61(0.58,0.62)$ & $731(689,749)$ \\
\hline & DLB & $0.61(0.56,0.65)$ & $703(692,734)$ \\
\hline & $A D$ & $0.59(0.56,0.61)$ & $734(699,752)$ \\
\hline \multirow[t]{3}{*}{ Corticopontine tract } & $\mathrm{CN}$ & $0.51(0.48,0.59)$ & $711(696,724)$ \\
\hline & DLB & $0.51(0.46,0.58)$ & $700(683,746)$ \\
\hline & $A D$ & $0.52(0.49,0.56)$ & $717(703,731)$ \\
\hline \multirow[t]{3}{*}{ Corpus callosum } & $\mathrm{CN}$ & $0.76(0.73,0.8)$ & $814(764,850)$ \\
\hline & DLB & $0.75(0.71,0.79)$ & $802(762,825)$ \\
\hline & $A D$ & $0.79(0.73,0.84)$ & $817(784,851)$ \\
\hline
\end{tabular}

Abbreviations: $A D=$ Alzheimer disease; $C N=$ cognitively normal; $D L B=$ dementia with Lewy bodies; DTI = diffusion tensor MRI; FA = fractional anisotropy; ILF = inferior longitudinal fasciculus; $\mathrm{MD}=$ mean diffusivity; $\mathrm{ROI}=$ region of interest; $\mathrm{SLF}=$ superior longitudinal fasciculus.

a Different from the $\mathrm{CN}$ group.

${ }^{\mathrm{b}}$ Different from DLB ( $p<0.05$ on pairwise Wilcoxon rank-sum/Mann-Whitney $U$ test).

${ }^{c}$ Different from both $\mathrm{CN}$ and DLB. ments in characterizing patients with $\mathrm{AD}$ and patients with DLB in bivariate logistic regression models. We found that elevated amygdala MD contributed (OR for 25-unit change in $\mathrm{MD}\left[\mathrm{OR}_{25}\right]$ 1.6; 95\% CI $1.0-2.4 ; p=0.04)$ to distinguishing patients with DLB from the CN group when included in a model along with GM density. In patients with $\mathrm{AD}, \mathrm{MD}$ contributed to the model for distinguishing patients with $\mathrm{AD}$ from $\mathrm{CN}$ when included with GM density in the hippocampus $\left(\mathrm{OR}_{25} 2.5 ; 95 \% \mathrm{CI}\right.$ $1.3-4.7 ; p=0.002)$ and the parahippocampal gyrus $\left(\mathrm{OR}_{25} 2.0 ; 95 \%\right.$ CI $\left.1.2-3.3 ; p=0.002\right)$. MD also contributed to the model for distinguishing patients with $\mathrm{AD}$ from $\mathrm{DLB}$ when included with GM density in the hippocampus $\left(\mathrm{OR}_{25} 1.9 ; 95 \%\right.$ CI 1.1-3.1; $p=0.01)$ and the parahippocampal gyrus $\left(\mathrm{OR}_{25}\right.$ 1.7; $95 \%$ CI $1.0-2.9 ; p=0.02)$.

FA was reduced $(p=0.01)$ and MD was elevated $(p=0.05)$ in the ILF of patients with DLB compared to CN. Higher MD was identified only in ILF of patients with DLB with visual hallucinations compared to the patients with DLB without visual hallucinations $(p=0.007)$. There were no associations between the ILF diffusivity measurements vs UPDRS scores and fluctuations in DLB $(p>0.05)$. Patients with $\mathrm{AD}$ had reduced FA and elevated MD in the fornix ( $p=0.06$ for $\mathrm{FA}$ and $p=0.01$ for $\mathrm{MD}$ ), posterior cingulum ( $p=0.06$ for FA and $p=$ 0.007 for $\mathrm{MD}$ ), and inferior longitudinal fasciculi ( $p=0.005$ for FA and $p=0.003$ for MD) compared to the $\mathrm{CN}$ group. No changes in diffusivity measurements were identified in the corpus callosum, SLF, and corticopontine tracts in patients with $\mathrm{AD}$ and patients with DLB (table 2).

DISCUSSION DTI findings in this study indicate that the diffusivity in the amygdala is elevated in patients with DLB. On the other hand, GM density in the amygdala was normal in DLB, which suggests that the pathologic mechanisms influencing amygdala diffusivity in DLB do not result in macroscopic loss of cortical tissue volume. This dissociation between the local diffusivity change and macroscopic loss of GM differs fundamentally between DLB and AD.

The amygdala is one of the cortical regions involved earliest and most severely with the LB pathology (figure 3). ${ }^{26,27} \mathrm{~A}$ common pathologic feature of this involvement is spongiosis or microvacuolation. ${ }^{28,29}$ Increased intravoxel incoherent motion of water molecules in the microvacuoles may be the underlying pathologic mechanism for the increased diffusivity in the amygdala. Another pathologic mechanism which may contribute to increased cortical diffusivity in neurodegenerative diseases is the loss of neuronal structure. However, neuronal loss in the cortex is not a prominent feature of LB 
Figure 3 Diffusivity changes that characterize

Alzheimer disease (AD) and

dementia with Lewy bodies (DLB)

follow pathologic progression

A

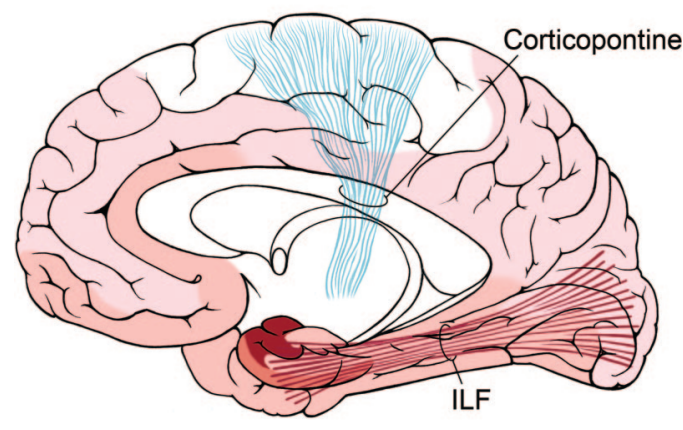

B

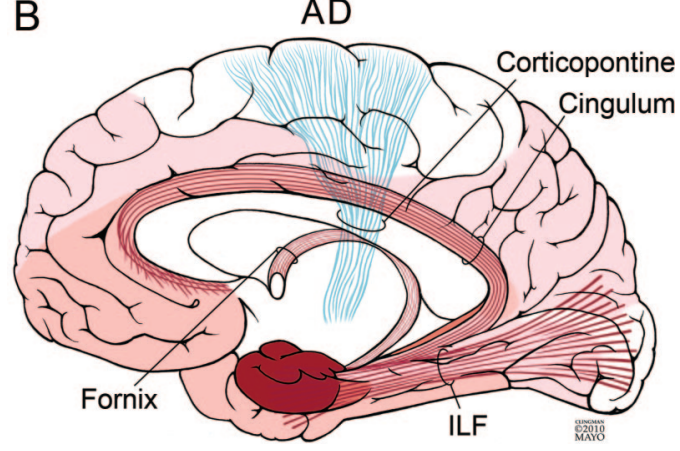

Distribution of Lewy body pathology (A) and neurofibrillary pathology of $A D(B)$ are illustrated (modified from Braak et al. ${ }^{26,37}$ ). (A) In the cerebral cortex, the earliest and most severe involvement with the Lewy body pathology is in the amygdala (shown in dark red). Lewy body pathology spreads to the temporal, occipital, and basal frontal association cortices as the disease progresses (shown in light red). In keeping with the topography of pathologic involvement, diffusivity changes in the amygdala and the temporo-occipital connections carried by the inferior longitudinal fasciculus (ILF) (shown in red) characterize the DTI abnormalities in DLB. The primary motor cortex (shown in white) and the corticopontine tracts (shown in blue) are generally spared and do not show DTI changes. (B) Hippocampus and the parahippocampal gyrus are the earliest regions to be involved with the neurofibrillary pathology of $A D$ (shown in dark red). The neurofibrillary pathology spreads to the temporal and parietal lobe association cortices as the disease progresses (shown in light red). Diffusivity changes in $A D$ follow the distribution of the neurofibrillary pathology and the associated neurodegeneration. The most significant DTI abnormalities are in the hippocampus and parahippocampal gyrus, and in the connecting tracts to these regions such as the ILF, cingulum, and fornix (shown in red). The primary motor cortex (shown in white) and the corticopontine tracts (shown in blue) are generally spared and do not show DTI changes (copyrighted and used with permission of Mayo Foundation for Medical Education and Research, all rights reserved).

disease, ${ }^{30,31}$ and amygdala GM density, which is an indirect measure of the neuronal density, ${ }^{32}$ was normal in patients with DLB in the current study and others from our group. ${ }^{24}$ Thus, elevated diffusivity in the absence of decreased GM density in the amygdala in DLB suggests that microvacuolation may be underlying the observed MD elevation in the amygdala of patients with DLB. We found a significant correlation between amygdala diffusivity and UPDRS scores in patients with DLB. However, it is unlikely that there would be a direct functional-structural relationship between amygdalar degeneration and parkinsonism. One explanation could be that the severity of LB pathology in the brain is associated with both increased amygdalar diffusivity and parkinsonism, thus producing an indirect association between amygdalar MD and symptom severity. Pathologic confirmation is required to understand the mechanisms underlying this association.

In patients with DLB, the directionality of diffusion measured with FA is decreased in the inferior longitudinal fasciculus, which projects from the occipital lobe visual association cortex to the medial and anterior temporal lobe and also carries the neuromodulatory backprojections from the amygdala to the visual cortex. ${ }^{33}$ Inferior longitudinal fasciculus diffusivity changes have been identified in a smaller group of patients with DLB. ${ }^{15}$ Based on the massive accumulation of $\alpha$-synuclein aggregates in the presynaptic terminal, and the reduction in synaptic activity markers, recent hypotheses suggest that primary pathway of neurodegeneration in DLB involves the presynaptic terminal. ${ }^{31,34}$ Therefore a disruption in the inferior longitudinal fasciculus measured with DTI in the current study may be related to a retrograde degeneration of the axonal projections that connect the amygdala and the visual association cortex (figure 3). The amygdalae are responsible for interpreting and providing emotional imprinting value to incoming visual information. Dysfunction of the visuo-amygdaloid pathway has been implicated in the visual misidentification and visual hallucinations. ${ }^{35,36}$ In the current study, diffusivity was significantly higher in the inferior longitudinal fasciculi of patients with DLB who were experiencing visual hallucinations compared to the patients with DLB who did not have this symptom, suggesting that visual hallucinations in DLB may be associated with the disruption of the temporo-occipital projections.

Diffusivity changes in the cortical GM of patients with $\mathrm{AD}$ were more widespread and severe than in patients with DLB, in agreement with a previous study in $\mathrm{AD}$ and DLB using a FLAIR-DTI sequence similar to our protocol in order to avoid partial volume averaging of cortical tissue with CSF. ${ }^{14}$ Patients with AD had increased diffusivity and reduced GM density in cortical regions that are typically involved with the neurofibrillary pathology such as the medial temporal lobe, posterior cingulate gyrus, precuneus, and the temporoparietal association cortices compared to both $\mathrm{CN}$ subjects and patients with DLB (figure 3). Since the topography of increased diffusivity and reduced GM 
density were similar in patients with $\mathrm{AD}$, an obvious question that emerged from this data was whether cortical diffusivity would provide any additional information over the cortical GM density in characterizing the neurodegenerative changes in $\mathrm{AD}$.

The addition of cortical diffusivity improved the ability of the logistic regression model with GM density to distinguish patients with $\mathrm{AD}$ from $\mathrm{CN}$ and from DLB only in the hippocampus and parahippocampal gyrus but not elsewhere in the cortex. Hippocampus and the parahippocampal gyrus are among the earliest and most severely involved regions with the neurodegenerative pathology in AD. ${ }^{37}$ Atrophy or reduced GM density has been shown to be highly correlated with the neuronal counts in these regions. ${ }^{32}$ The elevation in diffusivity may also be attributed to a loss of neuronal cell membranes that restrict the random motion of water molecules in the tissue, ${ }^{38}$ which may explain the similarities between the pattern of cortical diffusivity and GM density changes in $\mathrm{AD}$. However, our data indicate that hippocampal and parahippocampal gyrus diffusivity provided additional information over the GM density in characterizing the neurodegenerative changes in $\mathrm{AD}$. This is in agreement with the finding that hippocampal diffusivity improves the ability of hippocampal volumes to predict progression from amnestic mild cognitive impairment to $\mathrm{AD} .{ }^{39}$ It is possible that an additional pathologic process may be contributing to the elevated diffusivity in the medial temporal lobe regions severely affected with the neurodegenerative pathology of $\mathrm{AD}$ such as vacuolization. ${ }^{28,40}$ It is not obvious in which direction vacuolization might influence the tissue volume, but vacuolization should increase diffusivity, providing additional information over atrophy.

In patients with $\mathrm{AD}$, the directionality of diffusivity measured with FA was decreased and MD was elevated in the fornix, cingulum, and the inferior longitudinal fasciculus, which constitute the main limbic pathways connecting to the medial temporal lobe, in agreement with previous studies ${ }^{4-12}$ (figure 3).

A limitation of our study is the lack of pathologic confirmation in our subjects. Patients who meet clinical criteria for DLB often have some degree of $\mathrm{AD}$ pathology at autopsy and LB pathology may be present in some $\mathrm{AD}$ cases. Although we excluded subjects who met the clinical criteria for both AD and DLB, we acknowledge that some of our patients likely have mixed pathologies. However, on average clinically defined subjects with DLB should reflect imaging features of DLB more than $\mathrm{AD}$ and vice versa.

The differences in the topography of GM loss relative to the diffusivity change in $\mathrm{AD}$ compared to $\mathrm{DLB}$ imply fundamental differences in the pathologic mechanisms between the 2 diseases. In $\mathrm{AD}$, the $2 \mathrm{MRI}$ measures are topographically concordant, which can be explained by assuming that diffusivity change and GM loss are both due to neurodegeneration. In contrast, in DLB the amygdalar diffusivity change is not accompanied by loss of GM, implying a different pathologic mechanism such as vacuolization. The pattern of diffusivity changes in general reflected the underlying pathology, providing information independent of or complementary to the anatomic changes identified with structural MRI in both DLB and AD. The measures of diffusivity on DTI are valuable tools for characterizing the microstructural changes in neurodegenerative dementia and may be useful markers for early diagnosis and disease progression.

\section{AUTHOR CONTRIBUTIONS}

Statistical analysis was conducted by Stephen Weigand and Scott Przybelski.

\section{DISCLOSURE}

Dr. Kantarci receives research support from the NIH (K23 AG030935 [PI], P50 AG16574/P1 [PI], and R01 AG11378 [Co-]). Dr. Avula serves as a consultant for Medical Imaging Solutions. M.L. Senjem, A.R. Samikoglu, Dr. Zhang, S.D. Weigand, and S.A. Przybelski report no disclosures. Dr. Edmonson receives research support from the NIH (NIA R01 AG11378 [Co-I], NCRR UL1 RR 24150 [Co-I], NIBIB R01 EB00229 [Co-I], and NIMH R01 MH79261 [Co-I]); holds and has received royalties from Siemens on U.S. Patent 6,472,872; issued 2002: Real-time shimming of polarizing field in magnetic resonance system; and has received license fee payments for ACR phantom analysis software. Dr. Vemuri reports no disclosures. Dr. Knopman serves as an Associate Editor for Neurology ${ }^{\circledR}$; has served on data safety monitoring boards for Sanofi-Aventis, GlaxoSmithKline, and Eli Lilly and Company; is an investigator in clinical trials sponsored by Elan Corporation, Baxter International Inc., and Forest Laboratories, Inc.; and receives research support from the NIH (R01-AG023195 [PI], R01-AG11378 [CoI], P50 AG16574 [Co-I], U01 AG 06786 [Co-I], and R01 HL70825 $[\mathrm{Co}-\mathrm{I}])$. Dr. Ferman receives research support from the NIH (NIA R01AG015866 [PI]) and from the Mayo Clinic Alzheimer's Disease Research Center (P50 AG16574/P1 [Co-I]). Dr. Boeve has served as a consultant to GE Healthcare; receives royalties from the publication of Behavioral Neurology of Dementia (Cambridge Medicine, 2009); and receives research support from Myriad Genetics Inc., Cephalon, Inc., the NIH (P50 AG16574 [Co-I], UO1 AG06786 [Co-I, and RO1 AG15866 [Co-I]), the Alzheimer's Association, and the Center for Inherited Disease Research (U24 AG026395 [Co-I]). Dr. Petersen serves on scientific advisory boards for Elan Corporation, Wyeth, and GE Healthcare; receives royalties from the publication of Mild Cognitive Impairment (Oxford University Press, 2003); and receives research support from the NIH/NIA (U01 AG 06786 [PI], P50 AG 16574 [PI], U01 AG 024904 [Subcontract PI], and R01 AG11378 [Co-I]). Dr. Jack serves as a consultant for Elan Corporation; receives research support from Pfizer Inc., the NIH/NIA (R01-AG11378 [PI], P50-AG16574 [Co-I], and U01 AG024904-01 [Co-I]), and the Alexander Family Alzheimer's Disease Research Professorship of the Mayo Foundation; and holds stock in GE Healthcare and Johnson \& Johnson.

Received November 11, 2009. Accepted in final form February 23, 2010.

\section{REFERENCES}

1. Galasko D, Hansen LA, Katzman R, et al. Clinicalneuropathological correlations in Alzheimer's disease and related dementias. Arch Neurol 1994;51:888-895.

2. Schneider JA, Arvanitakis Z, Bang W, Bennett DA. Mixed brain pathologies account for most dementia cases in communitydwelling older persons. Neurology 2007;69:2197-2204.

3. Ulug AM, Moore DF, Bojko AS, Zimmerman RD. Clinical use of diffusion-tensor imaging for diseases causing 
neuronal and axonal damage. AJNR Am J Neuroradiol 1999;20:1044-1048.

4. Damoiseaux JS, Smith SM, Witter MP, et al. White matter tract integrity in aging and Alzheimer's disease. Hum Brain Mapp 2009;30:1051-1059.

5. Kantarci K, Jack CR, Jr., Xu YC, et al. Mild cognitive impairment and Alzheimer disease: regional diffusivity of water. Radiology 2001;219:101-107.

6. Medina D, DeToledo-Morrell L, Urresta F, et al. White matter changes in mild cognitive impairment and $\mathrm{AD}$ : a diffusion tensor imaging study. Neurobiol Aging 2006;27:663-672.

7. Mielke MM, Kozauer NA, Chan KC, et al. Regionallyspecific diffusion tensor imaging in mild cognitive impairment and Alzheimer's disease. Neuroimage 2009;46:47-55.

8. Ringman JM, O’Neill J, Geschwind D, et al. Diffusion tensor imaging in preclinical and presymptomatic carriers of familial Alzheimer's disease mutations. Brain 2007;130: 1767-1776.

9. Rose SE, Chen F, Chalk JB, et al. Loss of connectivity in Alzheimer's disease: an evaluation of white matter tract integrity with colour coded MR diffusion tensor imaging. J Neurol Neurosurg Psychiatry 2000;69:528-530.

10. Salat DH, Tuch DS, van der Kouwe AJ, et al. White matter pathology isolates the hippocampal formation in Alzheimer's disease. Neurobiol Aging 2010;31:244-256.

11. Stahl R, Dietrich O, Teipel SJ, Hampel H, Reiser MF, Schoenberg SO. White matter damage in Alzheimer disease and mild cognitive impairment: assessment with diffusion-tensor MR imaging and parallel imaging techniques. Radiology 2007;243:483-492.

12. Zhang Y, Schuff N, Jahng GH, et al. Diffusion tensor imaging of cingulum fibers in mild cognitive impairment and Alzheimer disease. Neurology 2007;68:13-19.

13. Bozzali M, Falini A, Cercignani M, et al. Brain tissue damage in dementia with Lewy bodies: an in vivo diffusion tensor MRI study. Brain 2005;128:1595-1604.

14. Firbank MJ, Blamire AM, Krishnan MS, et al. Diffusion tensor imaging in dementia with Lewy bodies and Alzheimer's disease. Psychiatry Res 2007;155:135-145.

15. Ota M, Sato N, Ogawa M, et al. Degeneration of dementia with Lewy bodies measured by diffusion tensor imaging. NMR Biomed 2009;22:280-284.

16. McKeith IG, Dickson DW, Lowe J, et al. Diagnosis and management of dementia with Lewy bodies: third report of the DLB Consortium. Neurology 2005;65:1863-1872.

17. Ferman TJ, Smith GE, Boeve BF, et al. Neuropsychological differentiation of dementia with Lewy bodies from normal aging and Alzheimer's disease. Clin Neuropsychol 2006;20:623-636.

18. Ferman TJ, Smith GE, Boeve BF, et al. DLB fluctuations: specific features that reliably differentiate DLB from AD and normal aging. Neurology 2004;62:181-187.

19. AASM. International Classification of Sleep Disorders 2: Diagnostic and Coding Manual. Chicago: American Academy of Sleep Medicine; 2005.

20. Kwong KK, McKinstry RC, Chien D, Crawley AP, Pearlman JD, Rosen BR. CSF-suppressed quantitative single-shot diffusion imaging. Magn Reson Med 1991;21:157-163.

21. Woods RP, Grafton ST, Holmes CJ, Cherry SR, Mazziotta JC. Automated image registration: I: general methods and intrasubject, intramodality validation. J Comput Assist Tomogr 1998;22:139-152.

22. Jiang H, van Zijl PC, Kim J, Pearlson GD, Mori S. DtiStudio: resource program for diffusion tensor computation and fiber bundle tracking. Comput Methods Programs Biomed 2006;81:106-116.

23. Tzourio-Mazoyer N, Landeau B, Papathanassiou D, et al. Automated anatomical labeling of activations in SPM using a macroscopic anatomical parcellation of the MNI MRI single-subject brain. Neuroimage 2002;15:273-289.

24. Whitwell JL, Weigand SD, Shiung MM, et al. Focal atrophy in dementia with Lewy bodies on MRI: a distinct pattern from Alzheimer's disease. Brain 2007;130:708-719.

25. Rothman KJ. No adjustments are needed for multiple comparisons. Epidemiology 1990;1:43-46.

26. Braak H, Del Tredici K, Rub U, de Vos RA, Jansen Steur EN, Braak E. Staging of brain pathology related to sporadic Parkinson's disease. Neurobiol Aging 2003;24:197-211.

27. Marui W, Iseki E, Nakai T, et al. Progression and staging of Lewy pathology in brains from patients with dementia with Lewy bodies. J Neurol Sci 2002;195:153-159.

28. Fujino Y, Dickson DW. Limbic Lobe microvacuolation is minimal in Alzheimer's disease in the absence of concurrent Lewy body disease. Int J Clin Exp Pathol 2008;1:369-375.

29. Jellinger KA. Lewy body-related alpha-synucleinopathy in the aged human brain. J Neural Transm 2004;111:1219-1235.

30. Gomez-Isla T, Growdon WB, McNamara M, et al. Clinicopathologic correlates in temporal cortex in dementia with Lewy bodies. Neurology 1999;53:2003-2009.

31. Klucken J, McLean PJ, Gomez-Tortosa E, Ingelsson M, Hyman BT. Neuritic alterations and neural system dysfunction in Alzheimer's disease and dementia with Lewy bodies. Neurochem Res 2003;28:1683-1691.

32. Bobinski M, de Leon MJ, Wegiel J, et al. The histological validation of post mortem magnetic resonance imagingdetermined hippocampal volume in Alzheimer's disease. Neuroscience 2000;95:721-725.

33. Catani M, Jones DK, Donato R, Ffytche DH. Occipitotemporal connections in the human brain. Brain 2003;126: 2093-2107.

34. Kramer ML, Schulz-Schaeffer WJ. Presynaptic alphasynuclein aggregates, not Lewy bodies, cause neurodegeneration in dementia with Lewy bodies. J Neurosci 2007; 27:1405-1410.

35. Ashtari M, Cottone J, Ardekani BA, et al. Disruption of white matter integrity in the inferior longitudinal fasciculus in adolescents with schizophrenia as revealed by fiber tractography. Arch Gen Psychiatry 2007;64:1270-1280.

36. Yamamoto R, Iseki E, Murayama N, et al. Investigation of Lewy pathology in the visual pathway of brains of dementia with Lewy bodies. J Neurol Sci 2006;246:95-101.

37. Braak H, Braak E. Neuropathological stageing of Alzheimerrelated changes. Acta Neuropathol 1991;82:239-259.

38. Sykova E, Vorisek I, Antonova T, et al. Changes in extracellular space size and geometry in APP23 transgenic mice: a model of Alzheimer's disease. Proc Natl Acad Sci USA 2005;102:479-484.

39. Kantarci K, Petersen RC, Boeve BF, et al. DWI predicts future progression to Alzheimer disease in amnestic mild cognitive impairment. Neurology 2005;64:902-904.

40. Smith TW, Anwer U, DeGirolami U, Drachman DA. Vacuolar change in Alzheimer's disease. Arch Neurol 1987;44:1225-1228. 